\title{
Rubus coreanus Miquel extract causes apoptosis of doxorubicin-resistant NCI/ADR-RES ovarian cancer cells via JNK phosphorylation
}

\author{
MIN KYOUNG KIM ${ }^{1}$, HYEONG SIM CHOI ${ }^{1}$, SUNG-GOOK CHO ${ }^{2}$, YONG CHEOL SHIN ${ }^{1}$ and SEONG-GYU KO ${ }^{1}$ \\ ${ }^{1}$ Department of Preventive Medicine, College of Korean Medicine, Kyung Hee University, \\ Seoul 130-701; ${ }^{2}$ Department of Biotechnology, Korea National University of Transportation, \\ Jeungpyeong-gun, Chungbuk 368-701, Republic of Korea
}

Received April 9, 2015; Accepted January 25, 2016

DOI: $10.3892 / \mathrm{mmr} .2016 .4996$

\begin{abstract}
Cancer cells can acquire an anticancer, drug-resistant phenotype following chemotherapy, which is tightly linked to cancer malignancy and patient survival rates. Therefore, the identification of options to treat chemotherapy-resistant cancer cells is an urgent requirement. Rubus coreanus Miquel (RCM) has long been used as a source of food. In addition, it has been reported that RCM has effective functions against particular diseases, including cancer and inflammation. In the present study, it was demonstrated that RCM extract caused the apoptotic cell death of doxorubicin-resistant NCI/ADR-RES ovarian cancer cells by phosphorylating c-Jun N-terminal kinase (JNK). The RCM-mediated reduction of cell viability showed no synergism with doxorubicin. In addition, ellagic acid and quercetin, which are phytochemicals found in RCM, also caused apoptosis of the NCI/ADR-RES cells. In subsequent investigations of the RCM-altered signaling pathway, RCM extract, ellagic acid and quercetin were found to commonly induce the phosphorylation of JNK and AKT. Additionally, the inhibition of JNK with SP600125 repressed the apoptotic cell death induced by RCM extract, ellagic acid and quercetin, and the inhibition of JNK appeared to switch apoptosis to necrosis. JNK inhibition also reduced the phosphorylation
\end{abstract}

Correspondence to: Professor Seong-Gyu Ko, Department of Preventive Medicine, College of Korean Medicine, Kyung Hee University, 1 Hoegi, Seoul 130-701, Republic of Korea

E-mail: epiko@khu.ac.kr

Professor Sung-Gook Cho, Department of Biotechnology, Korea National University of Transportation, 61 Daehakro, Jeungpyeong-gun, Chungbuk 368-701, Republic of Korea

E-mail: chosg@ut.ac.kr

Key words: Rubus coreanus Miquel, ellagic acid, quercetin, chemotherapy-resistance, doxorubicin, apoptosis, c-Jun N-terminal kinase of AKT, which was induced by RCM extract, ellagic acid and quercetin, suggesting that the phosphorylation of JNK is required for AKT phosphorylation in RCM-, ellagic acid- or quercetin-induced apoptotic cell death. Therefore, the data obtained in the present study led to the conclusion that RCM caused apoptosis of doxorubicin-resistant NCI/ADR-RES ovarian cancer cells via JNK phosphorylation, and suggested that RCM may be effective in the treatment of chemotherapy-resistant cancer cells.

\section{Introduction}

Cancer remains a serious disease, although its treatment options are well established. Chemotherapy is frequently used to treat cancer, and well-designed schedules for drug administrations are effective in treating cancer with fewer side-effects. However, certain cancer cells acquire a chemotherapy-resistant phenotype, resulting in difficulties in treatment (1-6). As the chemotherapy-resistant phenotype is tightly linked to the mechanism of epithelial-to-mesenchymal transition, chemotherapy-resistant cancer cells metastasize to distant organs (4-6). Therefore, the treatment of primary cancer cells and residual chemotherapy-resistant cancer cell populations are required to completely prevent cancer recurrence and metastasis (4-7).

Rubus coreanus Miquel (RCM), also known as Korean black raspberry, has long been used as a food and traditional medicine in Korea, Japan and China. Previous studies have reported that RCM is effective in the prevention and treatment of certain diseases, including cancer and inflammatory diseases (8-16). In cancer, RCM has been shown to repress the in vitro metastatic abilities of prostate cancer cells and cause apoptotic cell death $(9,15)$. However, the effect of RCM in other types of cancer has not been fully investigated, even in in vitro experimental settings, nor has the effect of RCM against anticancer drug-resistant cancer cells. Therefore, the role of RCM in cancer remains to be fully elucidated. In addition, although ellagic acid and quercetin, compounds found in RCM extract, are known to have anticancer properties (17-19), their effects in chemotherapy-resistant cancer cells remain to be elucidated. 
The present study aimed to investigate the effect of RCM on chemotherapy-resistant cancer cells. Doxorubicin-resistant NCI/ADR-RES ovarian cancer cells were used, as these cells are a well-established in vitro model system for investigations on chemotherapy-resistance (20). The current study hypothesized that RCM is effective in the treatment of chemotherapy-resistant cancer.

\section{Materials and methods}

Cell culture and drug preparation. The doxorubicin-resistant human ovarian cancer cell line, NCI/ADR-RES, was provided by Dr Hwa Jeong Lee (Ewha Womans University, Seoul, Korea). The NCI/ADR-RES cells were cultured in RPMI 1640 (WelGENE, Inc., Daegu, Korea) with $10 \%$ fetal bovine serum (Invitrogen; Thermo Fisher Scientific, Inc., Waltham , MA, USA) and $1 \%$ antibiotics-antimycotic solution (WelGENE, Inc.) in a humidified atmosphere with $5 \% \mathrm{CO}_{2}$ at $37^{\circ} \mathrm{C}$. The RCM extract was prepared by Hanpoong Pharmaceutical Company (Jeonju, Korea). The RCM was dissolved in $30 \%$ ethanol. Ellagic acid and quercetin were purchased from Tocris Biosciences (Boston, MA, USA) and Sigma-Aldrich (St. Louis, MO, USA), respectively. Ellagic acid was dissolved in distilled water at $25 \mathrm{mM}$, and quercetin was dissolved in dimethyl sulfoxide (DMSO; Sigma-Aldrich) at $30 \mathrm{mg} / \mathrm{ml}$.

Cell viability assay. An MTT assay was used to analyze the cell viabilities. The cells were seeded in flat-bottomed 96-well microplates at a density of $5 \times 10^{3}$ cells/well, and then treated with $0,12.5,25,50,100$ and $200 \mu \mathrm{g} / \mathrm{ml} \mathrm{RCM}$, with or without doxorubicin $(2 \mu \mathrm{g} / \mathrm{ml}$; Sigma-Aldrich) for $72 \mathrm{~h}$. Other groups of cells were treated with ellagic acid or quercetin at different concentrations $(0,10,20$ and $30 \mu \mathrm{g} / \mathrm{ml})$ for $72 \mathrm{~h}$. MTT solution (Sigma-Aldrich) was added to the medium and incubated at $37^{\circ} \mathrm{C}$ for $2 \mathrm{~h}$. Insoluble formazan was dissolved with DMSO and measured at $570 \mathrm{~nm}$ using a VersaMax ELISA microplate reader (Molecular Devices, LLC, Sunnyvale, CA, USA). The data examined was the average of three wells, and the experiment was independently repeated three times.

Analysis of cell apoptosis using flow cytometry. Annexin V detection assays were performed using Annexin V, Alexa Fluor ${ }^{\circledR} 488$ Conjugate (Molecular probes; Thermo Fisher Scientific, Inc.) and 7-aminoactinomycin D (Sigma-Aldrich). The NCI/ADR-RES cells were seeded at a density of $3 \times 10^{5}$ in a $60 \mathrm{~mm}$ plate. The cells were treated with RCM at concentrations of $0,50,100$ and $200 \mu \mathrm{g} / \mathrm{ml}$ for $24 \mathrm{~h}$ at $37^{\circ} \mathrm{C}$, or were treated with the ellagic acid or quercetin compounds at concentrations of $0,10,20$ and $30 \mu \mathrm{g} / \mathrm{ml}$. The cells were pretreated with $50 \mu \mathrm{M}$ of the JNK inhibitor, SP600125 (Sigma-Aldrich), for $1 \mathrm{~h}$ at $37^{\circ} \mathrm{C}$. The cells were then washed with phosphate-buffered saline (PBS), stained with Annexin V-fluorescein isothiocyanate (Thermo Fisher Scientific, Inc.) and 7-aminoactinomycin D, and then analyzed using flow cytometry.

Western blot analysis. The NCI/ADR-RES cells were seeded at a density of $8.5 \times 10^{5}$ in a $100 \mathrm{~mm}$ plate. The NCI/ADR-RES cells were treated with $\operatorname{RCM}(0,12.5,25,50,100,200 \mu \mathrm{g} / \mathrm{ml})$, ellagic acid $(0,10,20$ and $30 \mu \mathrm{g} / \mathrm{ml})$ or quercetin $(0,10,20$ and $30 \mu \mathrm{g} / \mathrm{ml}$ ) for $24 \mathrm{~h}$. Other groups of NCI/ADR-RES cells were treated with SP600125 at $50 \mu \mathrm{M}$ for $1 \mathrm{~h}$, and then treated with RCM $(100 \mu \mathrm{g} / \mathrm{ml})$, ellagic acid $(10 \mu \mathrm{g} / \mathrm{ml})$ or quercetin $(10 \mu \mathrm{g} / \mathrm{ml})$ for $24 \mathrm{~h}$ at $37^{\circ} \mathrm{C}$. Protein was obtained following cell lysis with radioimmunoprecipitation assay buffer (Biosesang, Inc., Seongnam, Korea). Subsequently, $15 \mu \mathrm{g}$ of protein was separated by standard 10 or $12 \%$ SDS-PAGE, and transferred onto polyvinylidene fluoride membranes. Mouse monoclonal anti-human B cell lymphoma-2 (Bcl-2; cat. no. sc-7382), mouse monoclonal anti-human $\mathrm{Bcl}-2$-associated $\mathrm{X}$ protein (BAX; cat. no. sc-7480), goat polyclonal anti-human cleaved caspase 9 (cat. no. sc-22182), rabbit polyclonal anti-human Raf-1 (cat. no. sc-133), mouse monoclonal anti-human p-JNK (cat. no. sc-6254), mouse monoclonal anti-human p-extracellular signal-regulated kinase (ERK)2 (cat. no. sc-7383), mouse monoclonal anti-human ERK2 (cat. no. sc-1647), goat polyclonal anti-human mammalian target of rapamycin (mTOR; cat. no. sc-1549), GSK3 $\beta$ and rabbit polyclonal anti-human p53 (cat. no. 6243) antibodies were purchased from Santa Cruz Biotechnology, Inc. (Santa Cruz, CA, USA) and used at a dilution of 1:1,000. Rabbit monoclonal anti-human Fas (cat. no. 4233), rabbit monoclonal anti-human Trail (cat. no. 3219), rabbit monoclonal anti-human cleaved caspase-8 (cat.no. 9496), rabbit polyclonal anti-human cleaved caspase-3 (cat. no. 9661), rabbit polyclonal anti-human poly (ADP-ribose) polymerase (PARP; cat. no. 9542), rabbit polyclonal anti-human p-cRaf (cat. no. 9421), mouse monoclonal anti-human JNK (cat. no. 3708), rabbit polyclonal anti-human AKT (cat. no. 9272), rabbit anti-human polyclonal phosphorylated (p)-AKT (ser473; cat. no. 9271), rabbit polyclonal anti-human p-mTOR (cat. no. 2971), rabbit polyclonal anti-human p-GSK3 $\beta$ (cat. no. 9332) and rabbit polyclonal anti-human p-p53 (cat. no. 9284) antibodies were purchased from Cell Signaling Technology, Inc. (Danvers, MA, USA) and used at a dilution of 1:1,000. Mouse monoclonal anti-human cytochrome $\mathrm{C}$ antibody was obtained from BD Biosciences (San Jose, CA, USA; cat. no. 556433) and used at a dilution of 1:1,000. Mouse monoclonal anti-human anti- $\alpha$-tubulin antibody (cat. no. T5168) was obtained from Sigma-Aldrich and used at a dilution of 1:100,000. The membranes were incubated at $4^{\circ} \mathrm{C}$ overnight with specific primary antibodies, and were washed three times in PBS with 0.01\% Tween (Sigma-Aldrich; PBST). The membranes were then incubated at room temperature for $1 \mathrm{~h}$ with horseradish peroxidase-conjugated goat anti-mouse (cat. no. 074-1806), goat anti-rabbit (cat. no. 474-1506) or rabbit anti-goat (cat. no. 14-13-06) secondary antibodies (KPL, Inc., Gaithersburg, MD, USA) used at dilutions of 1:10,000-1:50,000. Subsequently, the membranes were washed with PBST three times and the protein bands were visualized using an enhanced chemiluminescence system, EZ-Western Lumi Pico (Dogen-Bio, Seoul, Korea) and exposed to X-ray film (Agfa-Gevaert N.V., Mortsel, Belgium).

Statistical analysis. Microsoft Excel 2010 software (Microsoft Corporation, Redmond, WA, USA) was used for statistical analysis. Data are presented as the mean \pm standard deviation of at least three separate tests. The Student's t-test was used for single-variable comparisons and $\mathrm{P}<0.05$ was considered to indicate a statistically significant difference. 
A

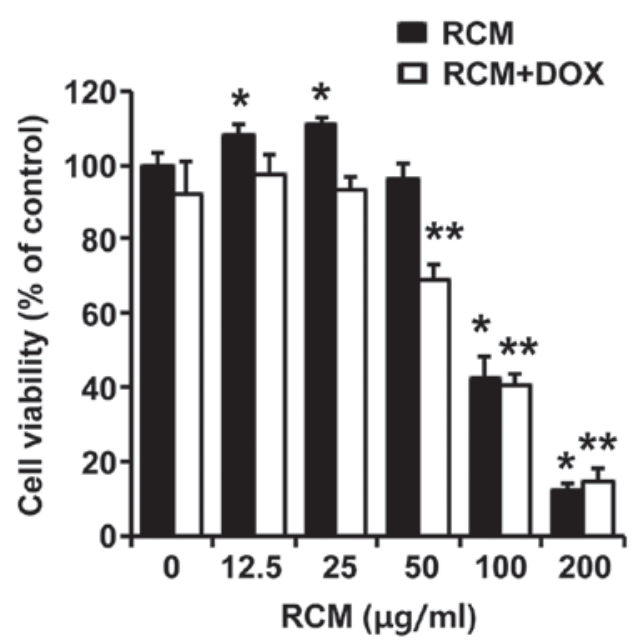

C

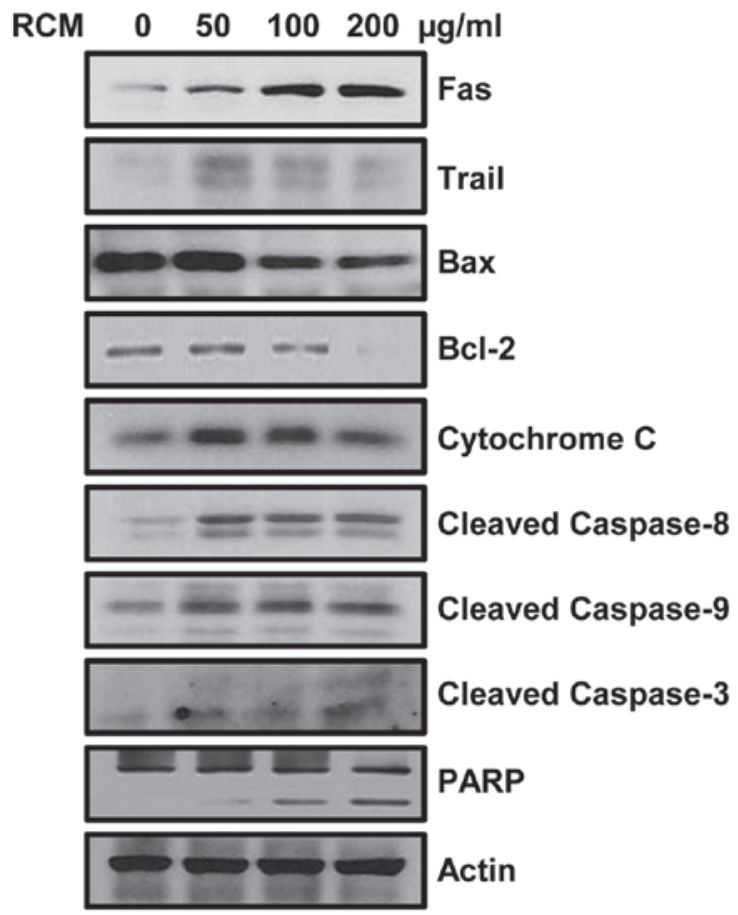

B

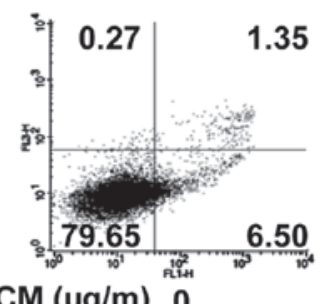

$\operatorname{RCM}(\mu \mathrm{g} / \mathrm{m}) \quad 0$

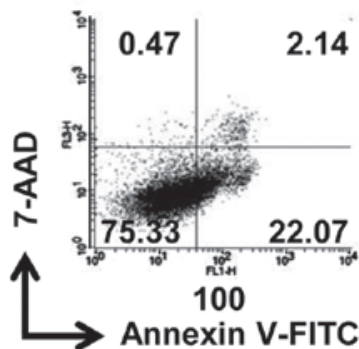

Annexin V-FITC

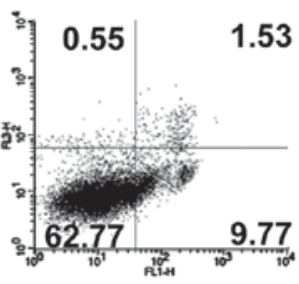

50

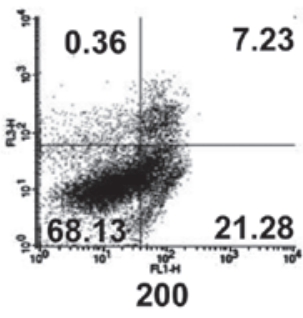

7.23
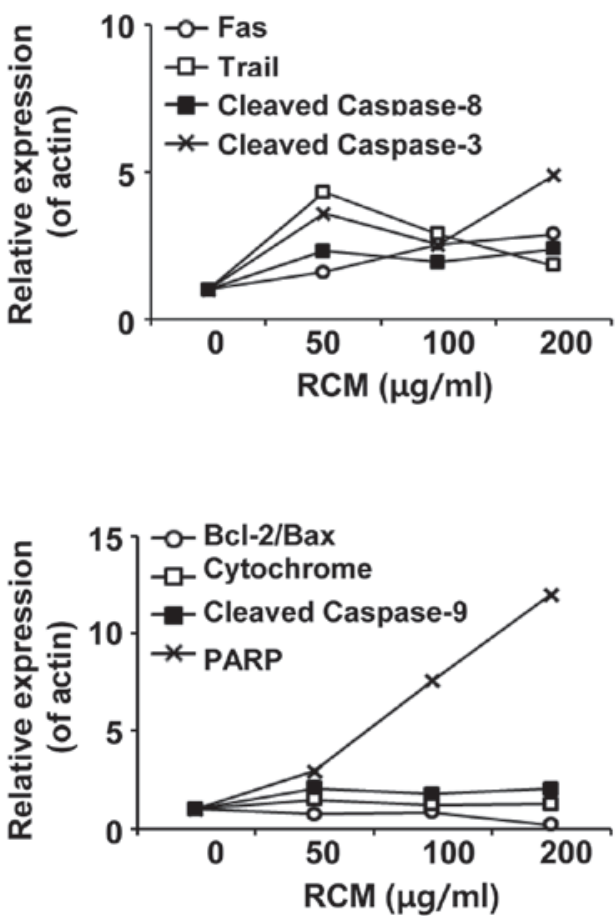

Figure 1. RCM induces apoptosis of NCI/ADR-RES cells. (A) MTT assay. Cells were treated with the indicated concentrations of RCM and $2 \mu \mathrm{g} / \mathrm{ml}$ of doxorubicin was added. Viability assays were performed $72 \mathrm{~h}$ following treatment. RCM at $100-200 \mu \mathrm{g} / \mathrm{ml}$ significantly reduced cell viability. Experiments were performed in triplicate and independently repeated at least three times. Data are presented as the mean \pm standard deviation for triplicate experiments. ${ }^{*} \mathrm{P}<0.05$ vs. RCM-untreated cells, ${ }^{* *} \mathrm{P}<0.05$ vs. doxorubicin-treated cells.. (B) Cells were treated with the indicated concentrations of $\mathrm{RCM}$ for $24 \mathrm{~h}$ and then subjected to Annexin V assays. RCM increases the numbers of apoptotic cells. (C) Cells were treated with RCM at the indicated concentrations for $24 \mathrm{~h}$ and then subjected to Western blot assays. Actin was detected as an internal control. Left panel, RCM induced apoptosis-associated proteins; right panel, data represent quantitative results for left panel. RCM, Rubus coreanus Miquel; FITC, fluorescein isothiocyanate; 7-AAD, 7-aminoactinomycin D; DOX, doxorubicin; PARP, poly (ADP-ribose) polymerase; Bcl-2, B cell lymphoma-2; BAX, Bcl-2-associated X protein.

\section{Results}

RCM causes apoptosis of doxorubicin-resistantovariancancer cells. To examine the effect of RCM in chemotherapy-resistant cancer, doxorubicin-resistant NCI/ADR-RES ovarian cancer cells were treated with RCM extract at different concentrations for $72 \mathrm{~h}$ and then subjected to cell viability assays. RCM at concentrations of 12.5 and $25 \mu \mathrm{g} / \mathrm{ml}$ slightly increased cell viability but cell viabiltiy was not affected at $50 \mu \mathrm{g} / \mathrm{ml}$. RCM at 100 and $200 \mu \mathrm{g} / \mathrm{ml}$ significantly reduced cell viability. No synergistic effect was observed when the cells were co-treated with RCM and doxorubicin (Fig. 1A). Therefore, RCM inhibited the viability of doxorubicin-resistant NCI/ADR-RES ovarian cancer cells.

Subsequently, the present study examined whether RCM caused apoptosis of the NCI/ADR-RES cells. In the Annexin V assays, it was found that RCM increased the numbers of apoptotic cells (Fig. 1B). Consistently, it was found that RCM altered the expression levels of apoptotic-associated proteins (Fig. 1C). Therefore, these data indicated that RCM caused 
A
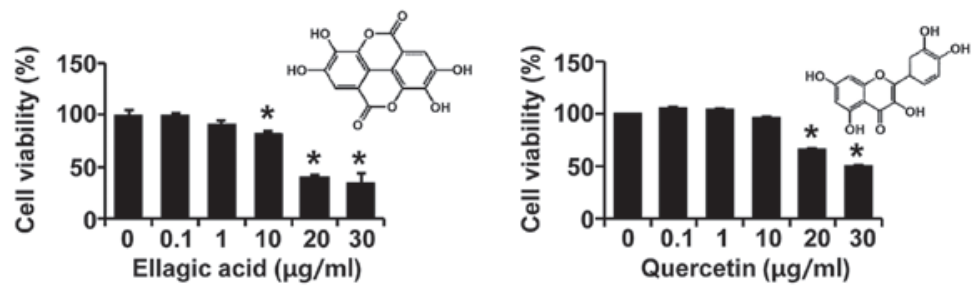

B
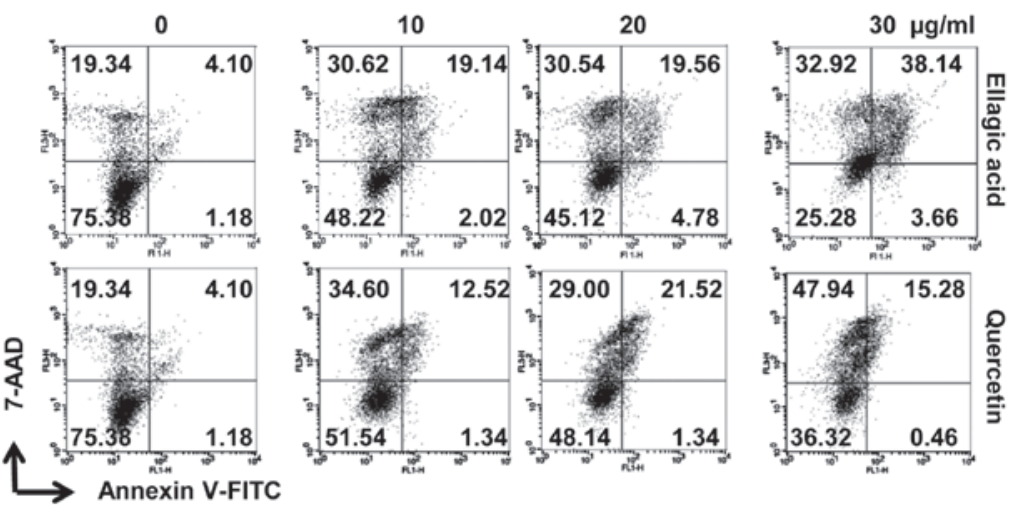

C

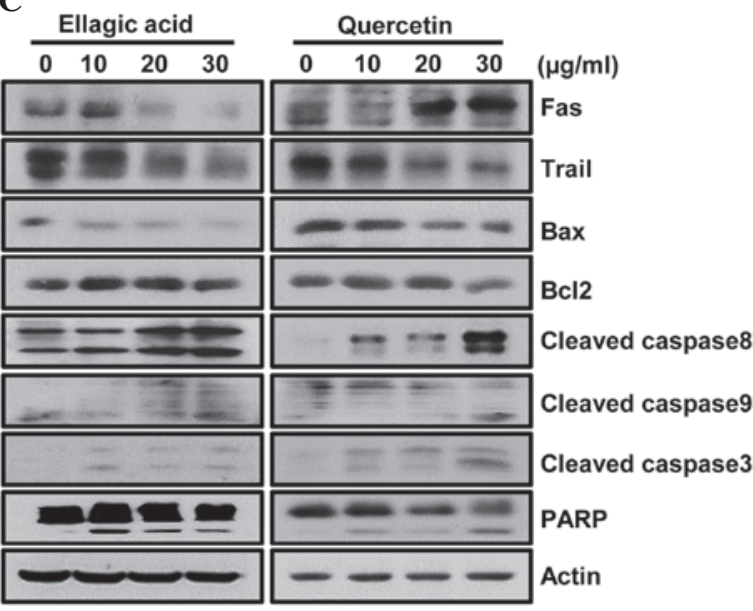

D
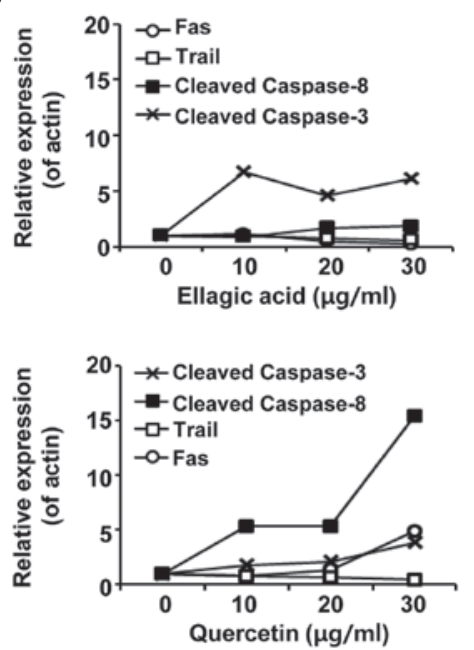
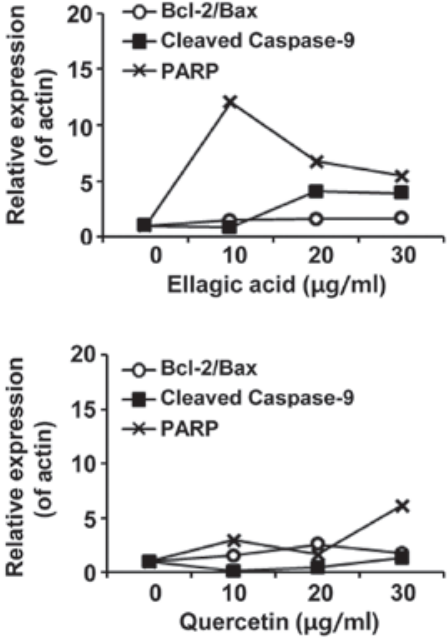

Figure 2. Ellagic acid and quercetin induce apoptosis of NCI/ADR-RES cells. (A) Cells were treated with ellagic acid or quercetin for 72 h, and subjected to MTT assays and (B) Annexin V assays. Cell numbers were analyzed using flow cytometry, with the numbers are indicated in the quadrants of the graphs. Ellagic acid and quercetin reduced cell viability and increased the numbers of apoptotic cells. Data are presented as the mean \pm standard deviation for triplicate experiments. ${ }^{*} \mathrm{P}<0.05$ vs. the control. (C) Cells were treated with ellagic acid or quercetin for $24 \mathrm{~h}$ and Western blot analysis was performed. Ellagic acid and quercetin induced apoptosis-associated protein expression. (D) Data represent quantitative results for western blot analysis. FITC, fluorescein isothiocyanate; 7-AAD, 7-aminoactinomycin D; PARP, poly (ADP-ribose) polymerase; Bcl-2, B cell lymphoma-2; BAX, Bcl-2-associated $\mathrm{X}$ protein.

apoptosis of chemotherapy-resistant ovarian cancer cells in a dose-dependent manner.

Ellagic acid and quercetin cause apoptosis of doxorubicin-resistant ovarian cancer cells. As RCM induced the apoptosis of NCI/ADR-RES cells, the present study further examined whether specific compounds found in RCM also have effects on apoptosis. When the NCI/ADR-RES cells were treated with the RCM-derived phytochemicals, ellagic acid or quercetin, at different concentrations for $72 \mathrm{~h}$, these compounds were found to reduce cell viabilities (Fig. 2A). In addition, ellagic acid and quercetin caused apoptotic cell death of the NCI/ADR-RES cells when the cells were treated with either phytochemical for $24 \mathrm{~h}$ (Fig. $2 \mathrm{~B}$ and C). Therefore, these data suggested that the effects of RCM on NCI/ADR-RES cells was, in part, due to the roles of these two compounds.

RCM induces the phosphorylation of JNK and AKT. As RCM and its compounds induced the apoptosis of NCI/ADR-RES cells, the present study further examined the effect of RCM on intracellular signaling molecules. RCM, ellagic acid and quercetin were observed to induce the phosphorylation of JNK and AKT, whereas each compound decreased Raf phosphorylation. The results also showed that ERK phosphorylation was not affected by RCM and ellagic acid, however, it was significantly increased by quercetin treatment (Fig. 3). Therefore, these data indicated that RCM and its compounds may cause apoptosis via JNK and/or AKT. 
A $\mathrm{RCM}$

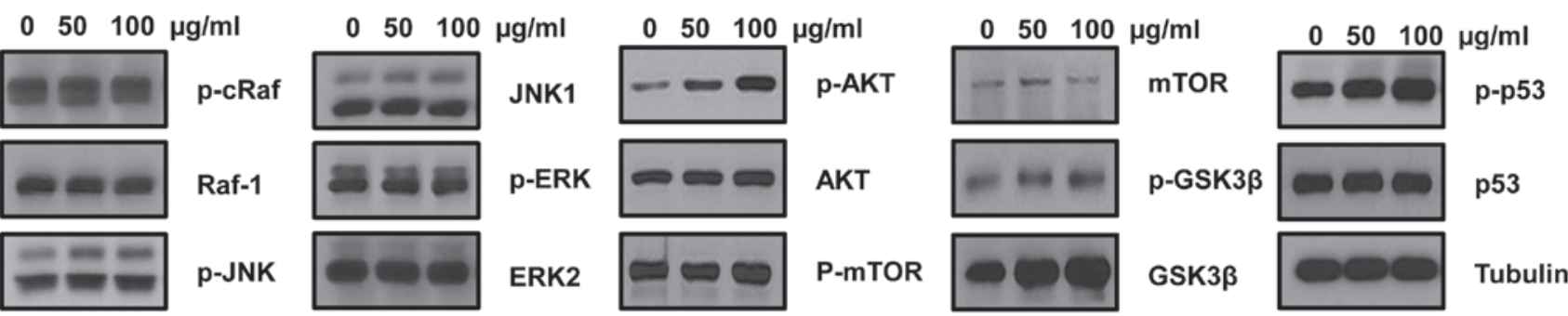

B
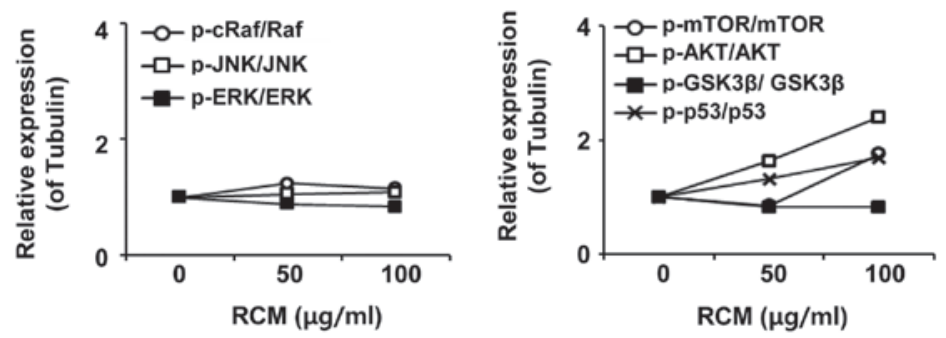

Ellagic acid

$\begin{array}{lllll}0 & 10 & 20 & 30 & \mu \mathrm{g} / \mathrm{ml}\end{array}$

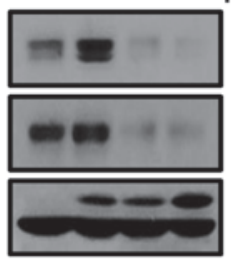

\section{p-cRaf \\ Raf-1 \\ p-JNK}

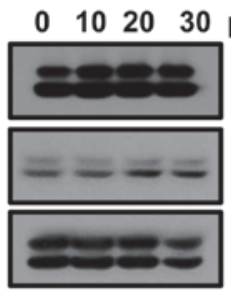

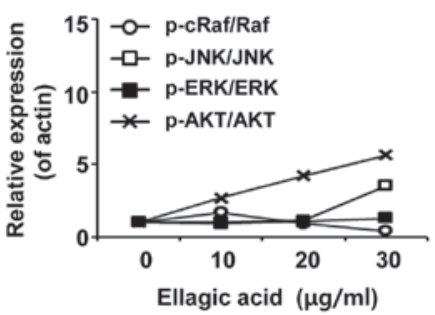

\section{Quercetin}

$\begin{array}{llll}0 & 10 & 20 & 30 \mu \mathrm{g} / \mathrm{ml}\end{array}$

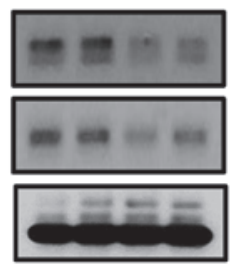

p-cRaf

Raf-1

p-JNK $\begin{array}{lllll}0 & 10 & 20 & 30 \mu \mathrm{g} / \mathrm{ml}\end{array}$

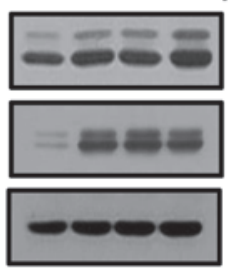

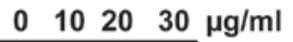
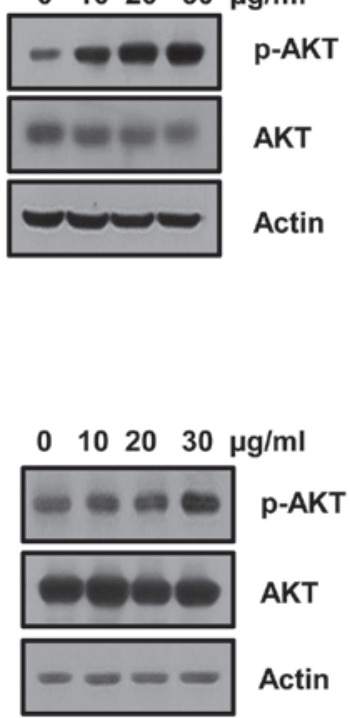

Actin
JNK1

p-ERK

ERK2

\section{JNK1 \\ p-ERK \\ ERK2}

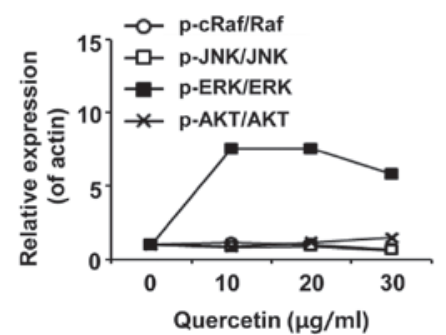

Figure 3. RCM activates JNK and AKT. The cells were treated with (A) RCM, (B) ellagic acid or (C) quercetin for $24 \mathrm{~h}$ and then subjected to Western blot analysis. Left panel, RCM, ellagic acid and quercetin induced the phosphorylation of JNK and AKT; right panel, data represent quantitative results. RCM, Rubus coreanus Miquel; JNK, c-Jun N-terminal kinase; ERK2, extracellular signal-regulated kinase 2; mTOR, mammalian target of rapamycin; p-, phosphorylated.

$J N K$ is required for RCM-induced apoptosis of NCI/ADR-RES cells. As RCM and its compounds were found to commonly induce the phosphorylation of JNK and AKT, the present study examined whether JNK was required for the effects of RCM on NCI/ADR-RES cells. When the cells were pretreated with the JNK inhibitor, SP600125, for $1 \mathrm{~h}$ and then treated with RCM, ellagic acid or quercetin for another $24 \mathrm{~h}$, JNK inhibition prevented apoptosis, but increased the numbers of necrotic cells (Fig. 4A). Consistently, JNK inhibition reduced PARP cleavage and inhibited the activation of caspases (Fig. 4B). Therefore, JNK phosphorylation appeared to be required for RCM-induced apoptotic cell death, and the inhibition of JNK phosphorylation may switch apoptosis to necrosis in RCM treatment.

Subsequently, the present study examined the association between JNK and AKT. As JNK phosphorylation was inhibited, the levels of AKT phosphorylation were also reduced (Fig. 4C). Therefore, these data indicated that RCM-induced JNK phosphorylation mediated the phosphorylation of AKT.

\section{Discussion}

In the present study, it was found that RCM and its compounds, ellagic acid and quercetin, caused JNK-dependent apoptotic cell death of doxorubicin-resistant NCI/ADR-RES ovarian cancer cells. Although RCM and its compounds have been reported to inhibit prostate cancer growth in vitro $(9,15)$, their inhibitory role in chemotherapy-resistant cancer has not been reported.

Treatment with RCM led to apoptotic cell death of the NCI/ADR-RES cells, which was found to occur in a dose-dependent manner. NCI/ADR-RES cells express high 
A
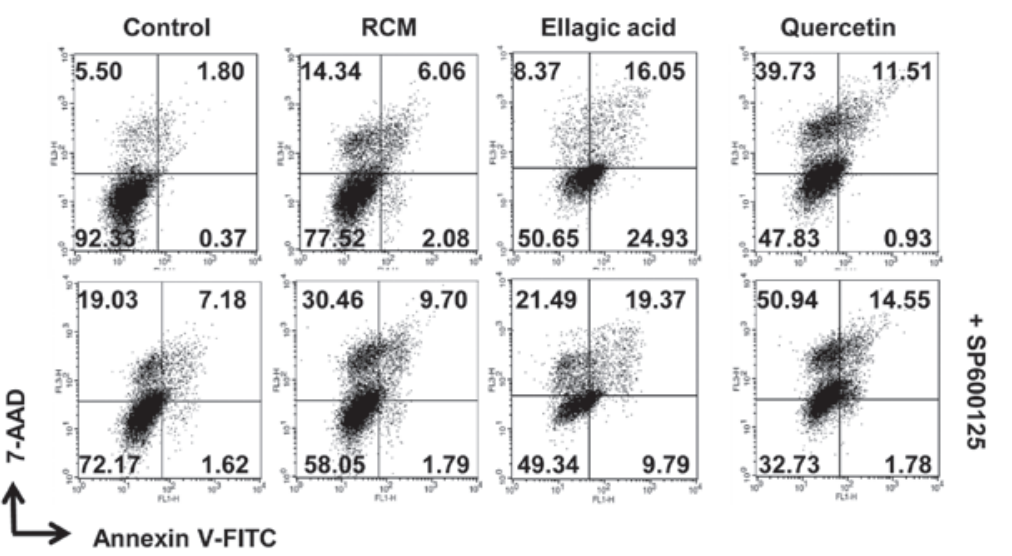

B
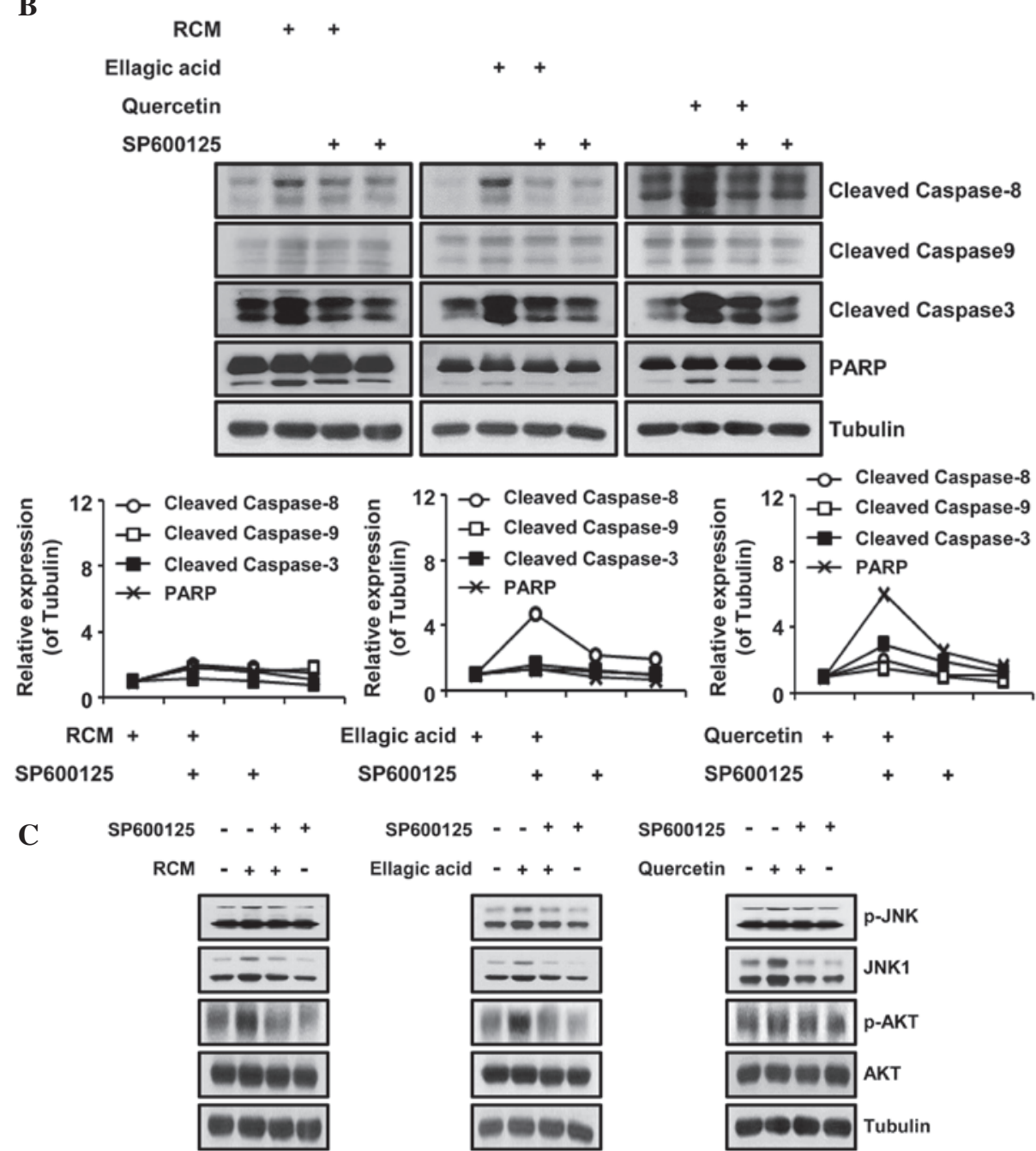

SP600125 -+++
Ellagic acid -++

SP600125 - - + +

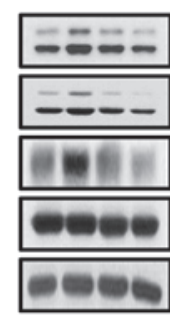

Quercetin - + -
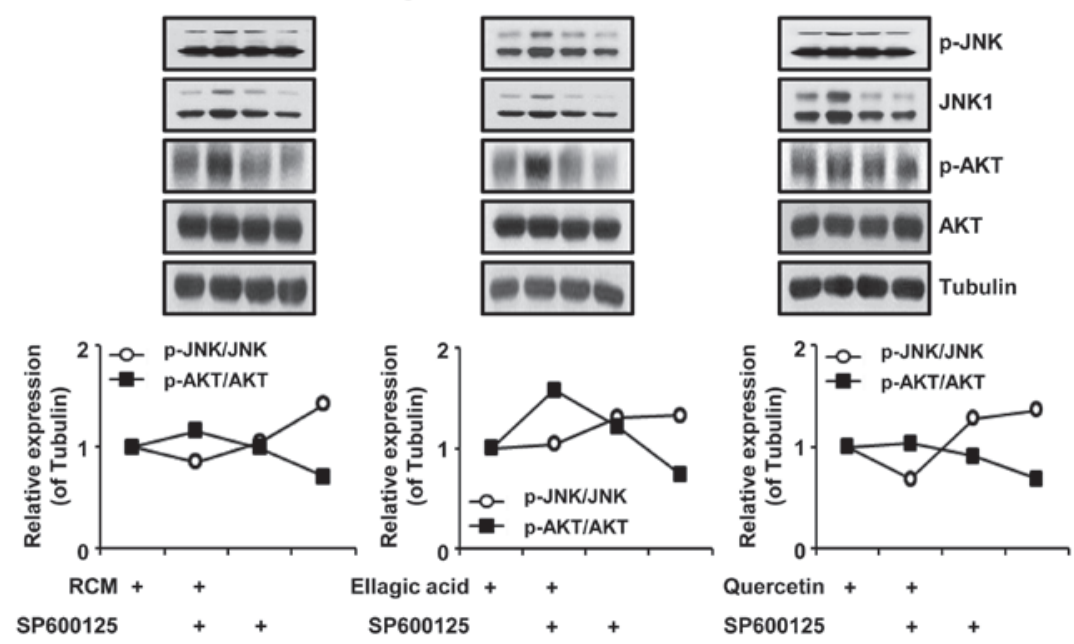

Figure 4. JNK inhibition inhibits RCM-induced apoptosis. (A) Cells were treated with RCM, ellagic acid or quercetin for $24 \mathrm{~h}$ and then subjected to Annexin $\mathrm{V}$ assays. Cell numbers are marked in the quadrants of the graphs. JNK inhibition prevented apoptosis by RCM, ellagic acid and quercetin, but increased the numbers of necrotic cells. (B and C) Cells were treated with the RCM, ellagic acid or quercetin, with or without SP600125 for $24 \mathrm{~h}$ and then subjected to Western blot analysis. Top panel, JNK inhibition reduced PARP cleavage, the activation of caspases, and AKT phosphorylation. Bottom panel, data represent quantitative results. RCM, Rubus coreanus Miquel; JNK, c-Jun N-terminal kinase; FITC, fluorescein isothiocyanate; 7-ADD, 7-aminoactinomycin D' PARP, poly (ADP-ribose) polymerase; ERK2, extracellular signal-regulated kinase 2; mTOR, mammalian target of rapamycin; p-, phosphorylated. 


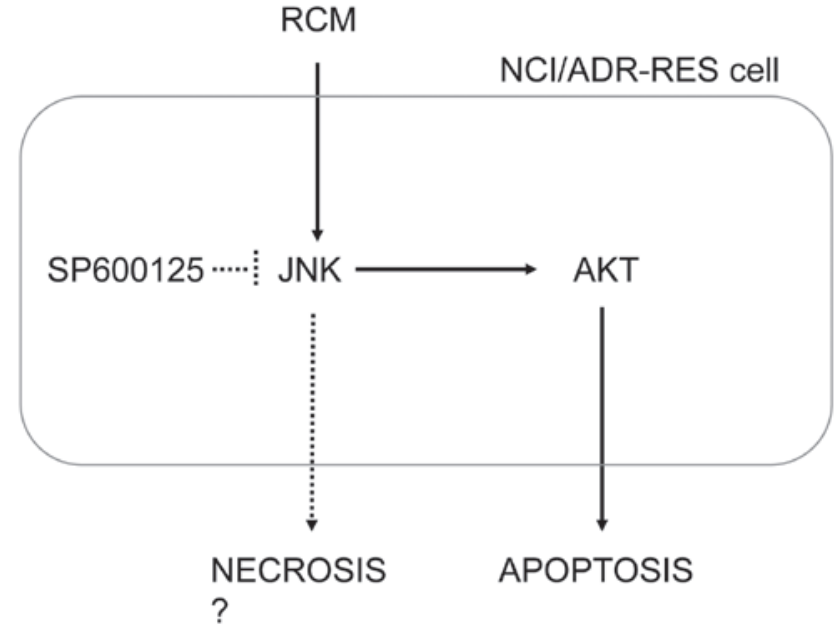

Figure 5. RCM induces apoptosis of NCI/ADR-RES cells via phosphorylating JNK and AKT. Schematic diagram showing that inhibition of the RCM-induced activation of JNK by SP600125 appeared to induce necrosis. RCM, Rubus coreanus Miquel; JNK, c-Jun N-terminal kinase.

levels of MDR1, thereby acquiring a chemotherapy-resistant phenotype (20). RCM appeared to inhibit MDR1 efflux and to regulate the expression of MDR1 (data not shown). However, RCM showed no synergism with doxorubicin. In assessing the potential synergistic effect, the highest dose of RCM $(200 \mu \mathrm{g} / \mathrm{ml})$ was effective in the inhibition of MDR1 efflux. As this highest concentration of RCM caused marked apoptosis in the experimental group, RCM-mediated apoptotic signaling was likely to precede any synergistic effect with doxorubicin.

As RCM, ellagic acid and quercetin were observed to inhibit cell viability to result in apoptosis, the present study hypothesized that these compounds, in part, mediated the effect of RCM on the NCI/ADR-RES cells. RCM and its compounds activated caspase- 8 , caspase- 9 and caspase- 3 , and reduced the level of BAX. In addition, whereas ellagic acid did not affect the level of Fas, RCM and quercetin increased Fas levels. The present study also confirmed that RCM affected mitochondrial membrane potential (data not shown). Thus, RCM appeared to activate the extrinsic and the intrinsic apoptotic pathways. Furthermore, RCM led to the accumulation of cells at the $\mathrm{S}$ phase (data not shown). Therefore, it was hypothesized that RCM has pleiotropic roles in cancer cells.

The present study also demonstrated that RCM, ellagic acid and quercetin uniquely activated JNK and AKT, although ellagic acid and quercetin differentially regulated Raf and ERK. In addition, the JNK inhibitor, SP600125, reduced the apoptotic effect of RCM. Thus, JNK mediated RCM-induced apoptosis. Consistently, the JNK inhibitor inhibited the apoptotic effects of ellagic acid and quercetin. Notably, it was further revealed that JNK inhibition induced necrotic cell death upon treatment with RCM or its compounds. Whereas the regulation of apoptosis and necrosis by JNK remains to be fully elucidated, these data consistently showed that JNK appeared to determine cell death, apoptosis and necrosis (Fig. 5). In addition, JNK inhibition decreased the phosphorylation of AKT induced by RCM and its compounds, indicating that JNK is upstream of AKT. Accordingly,
AKT inhibition also reduced RCM-induced apoptotic cell death, whereas the phosphorylation of JNK was not altered. In addition, increased necrosis was also found in the cells treated with AKT inhibitor (data not shown). A previous study revealed that JNK directly interacts with AKT in the rat ventricular myocytes (21). However, AKT phosphorylates JNK, which inhibits apoptosis in 293 and HeLa cells (22). Therefore, although a direct interaction between JNK and AKT was not examined, the present study suggested that the JNK-AKT pathway is crucial for the apoptosis of chemotherapy-resistant cancer cells.

In conclusion, the data obtained in the present study consistently demonstrated that RCM induced NCI/ADR-RES cell death. In addition, RCM-induced JNK activation determined the cell death as either apoptosis or necrosis. Although further information is required on the chemical compounds found in RCM extract, the compounds examined in the present study, ellagic acid and quercetin, were found to induce JNK-mediated apoptosis. Therefore, the results of the present study suggested that RCM may be beneficial for the treatment of chemotherapy-resistant cancer.

\section{Acknowledgements}

This study was supported by a grant from the Korean Medicine R\&D Project of the Ministry of Health and Welfare (grant no. B120014). This is part of the master thesis of Ms. Min Kyoung Kim.

\section{References}

1. Obenauf AC, Zou Y, Ji AL, Vanharanta S, Shu W, Shi H, Kong X, Bosenberg MC, Wiesner T, Rosen N, et al: Therapy-induced tumour secretomes promote resistance and tumour progression. Nature 520: 368-372, 2015.

2. Fu F, Nowak MA and Bonhoeffer S: Spatial heterogeneity in drug concentrations can facilitate the emergence of resistance to cancer therapy. PLoS Comput Biol 11: e1004142, 2015.

3. Oshimori N, Oristian D and Fuchs E: TGF- $\beta$ promotes heterogeneity and drug resistance in squamous cell carcinoma. Cell 160: 963-976, 2015.

4. Borst P: Cancer drug pan-resistance: Pumps, cancer stem cells, quiescence, epithelial to mesenchymal transition, blocked cell death pathways, persisters or what? Open Biol 2: 120066, 2012.

5. Meng F and Wu G: The rejuvenated scenario of epithelial-mesenchymal transition (EMT) and cancer metastasis. Cancer Metastasis Rev 31: 455-467, 2012.

6. Singh A and Settleman J: EMT, cancer stem cells and drug resistance: An emerging axis of evil in the war on cancer. Oncogene 29: 4741-4751, 2010.

7. Matsumoto K, Onda T and Yaegashi N: Pharmacotherapy for recurrent ovarian cancer: Current status and future perspectives. Jpn J Clin Oncol 45: 408-410, 2015.

8. Jung KA, Han D, Kwon EK, Lee CH and Kim YE: Antifatigue effect of Rubus coreanus Miquel extract in mice. J Med Food 10: 689-693, 2007.

9. Kim Y, Kim J, Lee SM, Lee HA, Park S, Kim Y and Kim JH: Chemopreventive effects of Rubus coreanus Miquel on prostate cancer. Biosci Biotechnol Biochem 76: 737-744, 2012.

10. Choi C, Lee H, Lim H, Park S, Lee J and Do S: Effect of Rubus coreanus extracts on diabetic osteoporosis by simultaneous regulation of osteoblasts and osteoclasts. Menopause 19: 1043-1051, 2012.

11. Kim SK,KimH,Kim SA,ParkHK and Kim W: Anti-inflammatory and anti-superbacterial activity of polyphenols isolated from black raspberry. Korean J Physiol Pharmacol 17: 73-79, 2013.

12. Im SE, Nam TG, Lee H, Han MW, Heo HJ, Koo SI, Lee CY and Kim DO: Anthocyanins in the ripe fruits of Rubus coreanus Miquel and their protective effect on neuronal PC-12 cells. Food Chem 139: 604-610, 2013. 
13. Chae HJ, Yim JE, Kim KA and Chyun JH: Hepatoprotective effects of Rubus coreanus miquel concentrates on liver injuries induced by carbon tetrachloride in rats. Nutr Res Pract 8: 40-45, 2014.

14. Choi MR, Lee MY, Hong JE, Kim JE, Lee JY, Kim TH, Chun JW, Shin HK and Kim EJ: Rubus coreanus Miquel ameliorates scopolamine-induced memory impairments in ICR mice. J Med Food 17: 1049-1056, 2014.

15. Kim Y, Lee SM and Kim JH: Unripe Rubus coreanus Miquel suppresses migration and invasion of human prostate cancer cells by reducing matrix metalloproteinase expression. Biosci Biotechnol Biochem 78: 1402-1411, 2014.

16. Shin JS, Cho EJ, Choi HE, Seo JH, An HJ, Park HJ, Cho YW and Lee KT: Anti-inflammatory effect of a standardized triterpenoid-rich fraction isolated from Rubus coreanus on dextran sodium sulfate-induced acute colitis in mice and LPS-induced macrophages. J Ethnopharmacol 158: 291-300, 2014.

17. Basu A, Nguyen A, Betts NM and Lyons TJ: Strawberry as a functional food: An evidence-based review. Crit Rev Food Sci Nutr 54: 790-806, 2014
18. Zhang HM, Zhao L, Li H, Xu H, Chen WW and Tao L: Research progress on the anticarcinogenic actions and mechanisms of ellagic acid. Cancer Biol Med 11: 92-100, 2014.

19. Miles SL, McFarland M and Niles RM: Molecular and physiological actions of quercetin: Need for clinical trials to assess its benefits in human disease. Nutr Rev 72: 720-734, 2014.

20. Liscovitch $M$ and Ravid D: A case study in misidentification of cancer cell lines: MCF-7/AdrR cells (re-designated NCI/ADR-RES) are derived from OVCAR-8 human ovarian carcinoma cells. Cancer Lett 245: 350-352, 2007.

21. Golden HB, Watson LE, Nizamutdinov D, Feng $\mathrm{H}$, Gerilechaogetu F, Lal H, Verma SK, Mukhopadhyay S, Foster DM, Dillmann WH and Dostal DE: Anthrax lethal toxin induces acute diastolic dysfunction in rats through disruption of the phospholamban signaling network. Int J Cardiol 168: 3884-3895, 2013.

22. Kim AH, Khursigara G, Sun X, Franke TF and Chao MV: Akt phosphorylates and negatively regulates apoptosis signal-regulating kinase 1. Mol Cell Biol 21: 893-901, 2001. 